\section{Enzalutamid bei mCRPC sicher}

Spricht ein metastasiertes, kastrationsresistentes Prostatakarzinom (mCRPC) nicht ausreichend auf eine Docetaxel-basierte Therapie an, ist Enzalutamid eine Option. Jetzt publizierten kanadische Wissenschaftler Daten zur Sicherheit dieser Behandlung.

n der Phase-III-Studie AFFIRM hatte sich die gute Wirksamkeit von Enzalutamid bei Patienten mit mCRPC, die zuvor Docetaxel erhalten hatten, erwiesen: Das mediane Gesamtüberleben der Patienten verlängerte sich von 13,6 auf 18,4 Monate (Hazard Ratio 0,63; $<<0,0001)$. 507 Patienten nahmen an einem ExpandedAccess-Programm vor der Zulassung von Enzalutamid teil. Die mehrfach vorbehandelten Patienten erhielten Enzalutamid (160 $\mathrm{mg} / \mathrm{Tag}$ ) bis die Krankheit voranschritt, inakzeptable Nebenwirkungen auftraten oder der Wirkstoff kommerziell erhältlich wurde. Am Tag 1, nach 4 und 12 Wochen und danach alle 12 Wochen wurden Nebenwirkungen erfasst. Als schwerwiegend wurden Ereignisse eingestuft, die lebensbedrohlich waren oder zum Tode führten, normale Lebensfunktionen nachhaltig beeinträchtigten oder stationär behandelt werden mussten. Das mediane Alter der Patienten lag bei 71 Jahren. $426(83,9 \%)$ von ihnen hatten einen Baseline-ECOG-Performancestatus von 1. Die meisten hatten außer Docetaxel noch weitere Therapien erhalten, am häufigsten Abirateron (76,1\%) oder Cabazitaxel (28,6\%).

Die mediane Therapiedauer in der Studie betrug 2,6 Monate (range 0,03-9,07). Die häufigsten Gründe für den Abbruch der Therapie waren die kommerzielle Erhältlichkeit von Enzalutamid (46,7\%) und ein Krankheitsprogress (33,7\%).

Insgesamt erlebten $88,2 \%$ der Patienten mindestens eine Nebenwirkung der Behandlung. Grad-1/2-Nebenwirkungen waren mit 45,4\% am häufigsten, gefolgt von Grad 3 (29,0\%), Grad 4 $(3,9 \%)$ und Grad 5 (9,9\%). Am häufigsten traten Fatigue (39,1\%), Nausea (22,7\%) und Appetitlosigkeit (14,8\%) auf. Schwerwiegende Nebenwirkungen gab es bei 143 Patienten $(28,2 \%)$, am häufigsten Krankheitsprogress (7,9\%), Pneumonie (2,0\%), Asthenie $(1,8 \%)$, Anämie $(1,6 \%)$ und Rückenschmerzen $(1,4 \%)$.

Bei 50 Patienten $(9,9 \%)$ führten Nebenwirkungen zum Tode. Häufigste Todesursache war ein Krankheitsprogress (7,7\%); darunter befanden sich zwei Patienten, die eine weitere zum Tode führende Nebenwirkung entwickelt hatten, eine gastrointestinale Perforation und eine Wundsepsis - beide gingen jedoch nicht auf das Konto der Therapie. Bei sieben weiteren Patienten traten (teilweise mehrere) zum Tode führende Ereignisse auf, die nicht unmittelbar mit der Therapie in Verbindung standen.

Fazit: Insgesamt, so Anthony M. Joshua und Kollegen, wurde die Therapie mit Enzalutamid von dieser schwierig behandelbaren Studienpopulation mit progressivem mCRPC gut vertragen. Das Sicherheitsprofil entsprach demjenigen der AFFIRM-Studie.

Kathrin von Kieseritzky

Joshua AM et al. Safety of Enzalutamide in Patients With Metastatic Castration-Resistant Prostate Cancer Previously Treated With Docetaxel: Expanded Access in North America. Prostate. 2015;75(8):836-44.

\section{Hier steht eine Anzeige.}

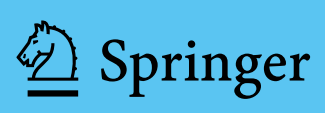

